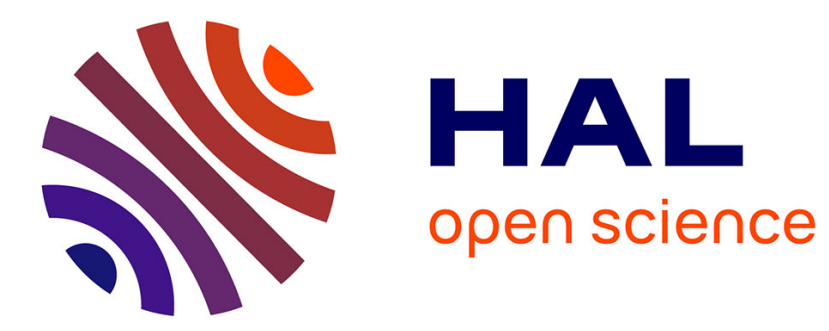

\title{
Magnétorhéomètre à bille
}

M. Adam, M. Delsanti, P. Pieransky, R. Meyer

\section{To cite this version:}

M. Adam, M. Delsanti, P. Pieransky, R. Meyer. Magnétorhéomètre à bille. Revue de Physique Appliquée, 1984, 19 (3), pp.253-264. 10.1051/rphysap:01984001903025300 . jpa-00245191

\section{HAL Id: jpa-00245191 https://hal.science/jpa-00245191}

Submitted on 1 Jan 1984

HAL is a multi-disciplinary open access archive for the deposit and dissemination of scientific research documents, whether they are published or not. The documents may come from teaching and research institutions in France or abroad, or from public or private research centers.
L'archive ouverte pluridisciplinaire HAL, est destinée au dépôt et à la diffusion de documents scientifiques de niveau recherche, publiés ou non, émanant des établissements d'enseignement et de recherche français ou étrangers, des laboratoires publics ou privés. 
Classification

Physics Abstracts

$06.90-07.90-80.40 \mathrm{~J}-61.40 \mathrm{~K}$

\title{
Magnétorhéomètre à bille
}

\author{
M. Adam, M. Delsanti \\ Laboratoire Léon Brillouin, CEN-Saclay, 91191 Gif sur Yvette, France \\ P. Pieransky
}

Laboratoire de Physique des Solides, Bât. 510, Université Paris XI, 91405 Orsay Cedex, France

et R. Meyer

Physics department, Brandeis Univ., Waltham, Massachusetts 02154, U.S.A.

(Reçu le 30 mai 1983, révisé le 28 octobre, accepté le 25 novembre 1983)

Résumé. - Nous présentons ici un nouveau rhéomètre qui permet de mesurer :

- la viscosité statique d'un fluide viscoélastique avec une précision de $2 \%$ sur une gamme allant de $3 \times 10^{-2}$ à $10^{5}$ poises $(\mathrm{P})$,

- le module élastique statique d'un solide viscoélastique avec une précision de $10 \%$ sur une gamme allant de $10^{-2}$ à $10^{4}$ dynes $/ \mathrm{cm}^{2}$,

- le temps de relaxation le plus long d'un corps viscoélastique avec une précision de $10 \%$ pour des temps de relaxation $\gtrsim 4 \times 10^{-2} \mathrm{~s}$.
Abstract. - We present a new rheometer which allows one to measure :
- for viscoelastic fluids, the static viscosity ranging from $3 \times 10^{-2}$ to $10^{5} \mathrm{P}$ within $2 \%$,
- for viscoelastic solid, the static elasticity ranging from $10^{-2}$ to $10^{4}$ dynes $/ \mathrm{cm}^{2}$ within $10 \%$
- the longest viscoelastic relaxation time within $10 \%$, in time range larger than $4 \times 10^{-2} \mathrm{~s}$.

\section{Introduction.}

Les propriétés viscoélastiques de cisaillement d'un matériau isotrope sont caractérisées si le module élastique de relaxation $G(t)$ ou sa viscosité dynamique $\eta(t)=\int_{0}^{t} G\left(t^{\prime}\right) \mathrm{d} t^{\prime}$ sont connus en fonction du temps. Les corps viscoélastiques peuvent être classés en deux catégories : les liquides viscoélastiques pour lesquels le module élastique statique $(G(t \rightarrow \infty))$ est nul, et les solides viscoélastiques qui ont un module statique fini. Sous l'effet des forces gravitationnelles les premiers coulent alors que les seconds ne coulent pas.

Pour mesurer les propriétés viscoélastiques d'un matériau, il existe un grand nombre d'appareils (pour une revue complète voir Refs. [1 et 2]). Le choix spécifique de l'appareil à utiliser dépend de la nature du corps à étudier et de la quantité que l'on désire connaître. Par exemple, la mesure de la viscosité statique d'un fluide viscoélastique peut être effectuée avec un viscosimètre à capillaire, Cannon Ubbelohde, à différents taux de cisaillement. Usuellement, on utilise des rhéomètres (cylindres coaxiaux ou côneplan par exemple) dont le fonctionnement est basé sur un mouvement périodique. L'échantillon est soumis à une déformation connue variant sinusoïdalement dans le temps. Une mesure des forces viscoélastiques exercées par le matériau permet de déterminer le module de relaxation complexe :

$$
G^{*}(\omega)=i \omega \int_{0}^{\infty} G(t) \mathrm{e}^{-i \omega t} \mathrm{~d} t
$$

Dans ces expériences les propriétés viscoélastiques sont sondées à un taux de cisaillement périodique dont l'amplitude est proportionnelle à la fréquence et à l'amplitude de la déformation. Qualitativement, la partie réelle de $G^{*}(\omega)$ correspond au module élastique de relaxation $G(t)$ à $t \approx 1 / \omega$. Une étude de $G^{*}(\omega)$ en fonction de la fréquence $\left(10^{-4} \mathrm{rad} . / \mathrm{s} \lesssim \omega \lesssim 10^{4} \mathrm{rad} . / \mathrm{s}\right)$ 
permet de remonter aux quantités suivantes : le module de relaxation $G(t)$, la viscosité et l'élasticité statiques.

Dans le cas du magnétorhéomètre à bille, il est imposé à l'éprouvette, contenant l'échantillon, un mouvement linéaire à vitesse constante. L'échantillon est soumis à un champ de taux de cisaillement constant dans le temps. Une mesure en régime stationnaire donne accès directement à la viscosité et à l'élasticité statiques. En régime transitoire, lorsqu'on supprime brusquement le mouvement, la force viscoélastique relaxe avec le temps avec une dépendance proportionnelle à $\int_{t}^{\infty} G\left(t^{\prime}\right) \mathrm{d} t^{\prime}$ (fluide viscoélastique). Une mesure de la force viscoélastique, permet alors de déterminer la relaxation qui est contrôlée par les temps viscoélastiques les plus longs. Les informations obtenues à partir du magnétorhéomètre à bille et d'un rhéomètre périodique sont donc différentes.

Le magnétorhéomètre à bille que nous décrivons ici (réalisé au CEN Saclay) présente l'avantage :

- d'appliquer des cisaillements et taux de cisaillement très faibles,

- d'éliminer les problèmes d'évaporation (l'échantillon est dans une éprouvette scellée),

- de thermaliser avec précision l'échantillon.

En outre cet appareil est d'un coût modeste.

\section{Théorie du fonctionnement.}

1.1 PRINCIPE DE L'APPAReIL. - Une bille magnétique (B.M.), immergée dans l'éprouvette (E.P.E.) contenant l'échantillon à analyser, est positionnée sur la partie extérieure de l'axe vertical d'une bobine B $(x=0$ voir Fig. 1). Dans cette région, le champ magnétique engendré par la bobine est non uniforme, la bille est donc soumise à une force magnétique qui est proportionnelle à l'intensité $I$ du courant circulant dans la bobine. L'intensité du courant est ajustée à une valeur telle que la force magnétique contrebalance la force effective de gravitation et toutes les forces qui peuvent être exercées sur la bille. Cet équilibre est réalisé grâce à une boucle d'asservissement dont la description est donnée ci-dessous. A l'aide d'une source lumineuse (S) et d'une lentille (L) l'image de la bille est formée dans le plan vertical $X, Y$. Les flux lumineux passant à travers le demi-plan supérieur $(X<0)$ et le demiplan inférieur $(X>0)$ sont envoyés respectivement sur les photodiodes $P_{1}$ et $P_{2}$. Le courant des photodiodes, montées en pont, est injecté dans un préamplificateur suivi d'un régulateur à action proportionnelle différentielle et intégrale (PID), qui pilote le générateur de courant de la bobine.

Pour déterminer les propriétés viscoélastiques de l'échantillon, il est imposé à l'éprouvette un déplacement uniforme colinéaire à l'axe de la bobine, la bille est alors soumise à l'effet d'une force viscoélastique. Par exemple, si l'échantillon est un liquide

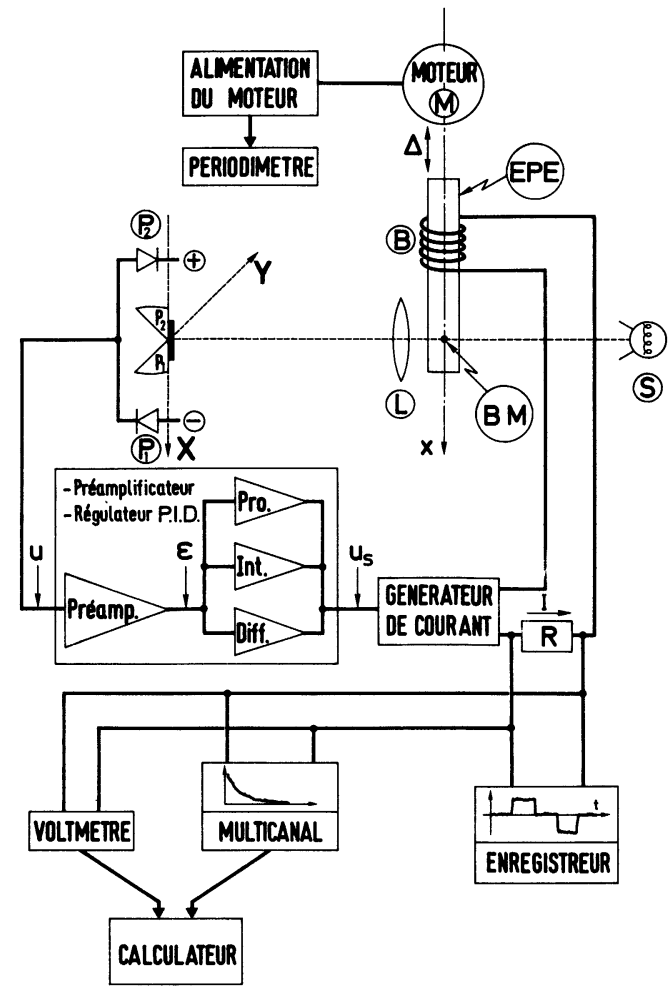

Fig. 1. - Bloc diagramme du magnétorhéomètre à bille. Pour la description et la signification des différents symboles voir les paragraphes $1.1,2.2,2.3$ et 2.5 .

[Block diagram of the magnetorheometer. A description of the apparatus and the symbols used is given in sections 1.1, 2.2, 2.3 and 2.5.]

Newtonien cette force est une force de frottement proportionnelle à la vitesse de déplacement de l'éprouvette et à la viscosité $\eta$ du liquide. Ceci induit une variation de l'intensité du courant circulant dans la bobine proportionnelle à la force de frottement. Moyennant une calibration à l'aide d'un liquide de viscosité connue, la valeur absolue de la viscosité est déterminée par les mesures de la variation de l'intensité du courant en fonction de la vitesse de déplacement de l'éprouvette.

1.2 FonCtion D'APPAREIL ET PRINCIPE DE MESURE. Lorsque l'éprouvette est au repos la bille de masse $m$ est soumise à une force magnétique $f_{0 \mathrm{~m}}$ qui contrebalance la force effective de gravitation $f_{\mathbf{g}}$, la bille est positionnée en $x=0$.

Lorsque l'éprouvette est soumise à un déplacement connu $\Delta(t)$, il s'exerce sur la bille les forces supplémentaires suivantes :

(a) la force d'inertie, $m \ddot{x}$,

(b) la force viscoélastique,

(c) la force de rappel magnétique $f_{\mathrm{m}}(t)$ qui contrebalance à tout instant les forces $(a)$ et $(b)$.

La force viscoélastique $(b)$ dépend du mouvement relatif de la bille par rapport à l'éprouvette $(x(t)-\Delta(t))$. 
Pour des raisons de commodité la force viscoélastique est décomposée en deux :

- la force viscoélastique $f_{\mathrm{v}}(t)$ due au mouvement propre de la bille $(x(t))$ qui dépend des conditions d'asservissement,

- la force viscoélastique externe $f_{\mathrm{e}}(t)$ qui dépend du déplacement $\Delta(t)$ imposé à l'éprouvette par l'expérimentateur.

La force viscoélastique externe, pour de faibles cisaillements et taux dẹ cisaillements, est reliée à la vitesse de déplacement $\dot{\Delta}(t)$ par une relation de convolution [2,3] (voir $\S 1.2 .1 .2$ ) :

$$
f_{\mathrm{e}}(t)=\mu(t) * \dot{\Delta}(t)
$$

où $\mu(t)$ est une constante de rappel généralisée qui est proportionnelle au module de relaxation viscoélastique $G(t)$ de l'échantillon.

Dans le cas d'un asservissement "idéal " la bille reste à une position fixe $(x(t)=0)$ donc la force d'inertie $m \ddot{x}$ et la force viscoélastique $f_{v}$ sont nulles. La force de rappel magnétique $f_{\mathrm{m}}(t)$ est alors à tout instant une réplique de la force viscoélastique externe $f_{\mathrm{e}}(t)$. La connaissance de $f_{\mathrm{m}}(t)$ et $\Delta(t)$ permet de remonter au module de relaxation de l'échantillon. Dans le cas d'un asservissement réel ni la force d'inertie ni la force $f_{\mathrm{v}}$ ne sont nulles. Le système étant linéaire (pour de faibles forces extérieures) et invariant par translation dans le temps, les quantités $f_{\mathrm{m}}(t)$ et $f_{\mathrm{e}}(t)$ sont reliées par une relation de convolution :

$$
-f_{\mathrm{m}}(t)=W(t) * f_{\mathrm{e}}(t),
$$

où $W(t)$ est la fonction d'appareil du magnétorhéomètre. Expérimentalement, la fonction d'appareil ne peut pas être déterminée car il est impossible d'engendrer une force extérieure percussionnelle sans perturber le système d'asservissement et sans changer les conditions expérimentales sous lesquelles sont effectuées les mesures viscoélastiques. Aussi il est nécessaire d'établir la structure de $W(t)$ et de déterminer les constantes de temps caractéristiques d'appareillage. Nous donnons dans ce chapitre la structure des forces, ensuite la fonction d'appareil et les conditions expérimentales de mesure.

\subsubsection{Structure des forces.}

1.2.1.1 Force de rappel magnétique. - Dans la configuration en boucle(Fig. 1) une variation de position $x$ de la bille induit une variation de la force magnétique :

$$
f_{\mathrm{m}}(t)=-K\left[\alpha x(t)+\tau_{\mathrm{D}} \dot{x}(t)+\frac{1}{\tau_{\mathrm{i}}} \int_{-\infty}^{t} x\left(t^{\prime}\right) \mathrm{d} t^{\prime}\right]
$$

$\dot{x}(t)$ est la vitesse de déplacement de la bille [4], $K$ est une constante de rappel proportionnelle au gain $\alpha_{0}$ du préamplificateur (pour plus de détail voir Eq. (22)). Les coefficients caractéristiques du régulateur PID parallèle sont :

- $\alpha$ le gain de l'action proportionnelle
- $\tau_{\mathrm{D}}$ la constante de temps de l'action différentielle - $\tau_{\mathrm{i}}$ la constante de temps de l'action intégrale.

Dans l'expression (3) il est supposé que les temps de réponse des photodiodes, du générateur de courant et de la bobine magnétique sont négligeables. Sous une forme plus condensée l'expression (3) s'écrit :

$$
f_{\mathrm{m}}(t)=A(t) * x(t) .
$$

$A(t)$ représente la fonction d'appareil de la boucle d'asservissement :

$$
A(t)=-K\left[\alpha \delta(t)+\tau_{\mathrm{D}} \dot{\delta}(t)+\frac{1}{\tau_{\mathrm{i}}} \theta(t)\right],
$$

$\delta(t)$ et $\theta(t)$ sont respectivement la fonction de Dirac et la fonction échelon.

1.2.1.2 Forces viscoélastiques. - Les forces viscoélastiques s'exerçant sur la bille sont proportionnelles à la contrainte effective de cisaillement $\sigma$ moyennée spatialement à la surface de la bille [2] :

$$
f_{\mathrm{e}}+f_{\mathrm{v}} \propto-r^{2} \sigma,
$$

$r$ représente le rayon de la bille. Très généralement la contrainte de cisaillement est à un instant $t$, le résultat de l'application des taux de cisaillement $\dot{s}$ antérieurs :

$$
\sigma(t)=\int_{-\infty}^{t} G\left(t-t^{\prime}\right) \dot{s}\left(t^{\prime}\right) d t^{\prime}
$$

$G(t)$ est le module de relaxation. Cette relation décrit un comportement viscoélastique linéaire et n'est valable qu'à des cisaillements et taux de cisaillement faibles. Dans notre cas $\dot{s}$ est un taux de cisaillement effectif proportionnel à la vitesse relative entre la bille et l'échantillon :

$$
\dot{s}(t) \propto(\dot{x}(t)-\dot{\Delta}(t)) / r .
$$

$\dot{\Delta}(t)$ représente la vitesse de déplacement de l'éprouvette. Les forces viscoélastiques s'expriment comme suit :

$$
\begin{aligned}
f_{\mathrm{v}}(t)+f_{\mathrm{e}}(t)=-\mu(t) *(\dot{x}(t) & -\dot{\Delta}(t))= \\
= & -\dot{\mu}(t) *(x(t)-\Delta(t))
\end{aligned}
$$

avec

$$
\mu(t)=\phi r G(t) \theta(t)
$$

$\mu(t)$ est une constante de rappel généralisée, $\phi$ est un facteur numérique qui ne dépend que de la géométrie de l'expérience à faibles cisaillement et taux de cisaillement.

Dans la suite du paragraphe, dans un but de simplification, nous ne ferons des commentaires que sur $f_{\mathrm{e}}$. Pour un fluide Newtonien [2], nous avons :

$$
\begin{aligned}
& G(t)=\eta \delta(t) \\
& \mu(t)=\phi \eta r \delta(t) .
\end{aligned}
$$


En portant (10) dans (9) nous retrouvons la loi de Stokes : $f_{\mathrm{e}}(t)=\phi \eta r \dot{\Delta}(t) ; \phi$ tend vers $6 \pi$ lorsque le rayon de la bille est petit par rapport aux dimensions de l'échantillon [1]. Pour un corps purement élastique, nous avons $[2,5]$ :

$$
\begin{aligned}
& G(t)=G_{\infty} \theta(t) \\
& \mu(t)=\phi r G_{\infty} \theta(t) .
\end{aligned}
$$

En portant (11) dans (9) nous trouvons que la force $f_{\mathrm{e}}$ est directement proportionnelle au module de cisaillement $G_{\infty}: f_{\mathrm{e}}(t)=\phi G_{\infty} r \Delta(t)$.

1.2.2 Fonction d'appareil et principe de mesure. En utilisant les formes explicites des forces (Eqs. (4), (5) et (9)) et la loi fondamentale de la dynamique :

$$
m \ddot{x}(t)=f_{\mathrm{m}}(t)+f_{\mathrm{v}}(t)+f_{\mathrm{e}}(t),
$$

nous trouvons que la position de la bille est reliée à la force extérieure par :

$$
x=\Omega * f_{\mathrm{e}}
$$

$\Omega$ est l'opérateur inverse de $m \ddot{\delta}+\dot{\mu}-A$. D'après la définition de la fonction d'appareil (Eq. (2)) et les équations (4) et (12a), nous obtenons :

$$
W=A * \Omega \text {. }
$$

La fonction d'appareil $W$ dépend des coefficients caractéristiques de l'asservissement $K, \alpha, \tau_{\mathrm{D}}$ et $\tau_{\mathrm{i}}$ (voir $\S 1.2 .1$ ) de la masse de la bille et des propriétés viscoélastiques de l'échantillon $\dot{\mu}$. Pour déterminer la structure de $W(t)$ il est nécessaire d'utiliser la transformée de Laplace de $W(t): \hat{W}(p)$ (voir Appendice). Il apparaît que les constantes de temps caractéristiques d'appareillage sont : $\tau_{\mathrm{m}}=\sqrt{m / K}, \tau_{\mathrm{v}}=\phi \eta r / K, \tau_{\mathrm{i}}$ et $\tau_{\mathrm{D}}$ les constantes de temps de différentiation et d'intégration du régulateur P.I.D. On peut remarquer aussi que si la constante de rappel tend vers l'infini $\left(\lim _{\boldsymbol{K} \rightarrow \infty} \hat{W}(p)=1\right)$ la fonction d'appareil tend vers une fonction de Dirac : on a alors un asservissement « idéal ".

La force viscoélastique extérieure est engendrée par un mouvement de l'éprouvette à vitesse constante $v$ pendant un temps $t_{0}$. En portant la vitesse de déplacement de l'éprouvette : $\dot{\Delta}(t)=v\left[\theta(t)-\theta\left(t-t_{0}\right)\right]$ dans l'expression (9), il vient pour la force viscoélastique extérieure :

$$
f_{\mathbf{e}}(t)=\phi r v \int_{0}^{t} G\left(t^{\prime}\right) \mathrm{d} t^{\prime}
$$

lorsque l'éprouvette est en mouvement $t \leqslant t_{0}$,

$$
f_{\mathrm{e}}(t)=\phi r v \int_{t-t_{0}}^{t} G\left(t^{\prime}\right) \mathrm{d} t^{\prime},
$$

lorsque le déplacement de l'éprouvette est arrêté $t>t_{0}$.
1.2.2.1 Mesures en régime stationnaire. - La mesure en régime stationnaire est par définition une mesure indépendante du temps. Elle est donc nécessairement effectuée dans une gamme de temps grande en comparaison des constantes de temps d'appareillage et du temps $\tau$ de relaxation viscoélastique le plus long de l'échantillon. En régime stationnaire la force de rappel magnétique $f_{\mathrm{m}}$ contrebalance la force viscoélastique extérieure $f_{\mathrm{e}}$. Les mesures en régime stationnaire permettent de déterminer les caractéristiques viscoélastiques statiques ( $\eta$ et $G_{\infty}$ ) de l'échantillon :

- Cas d'un fluide viscoélastique.

Lorsque l'éprouvette est déplacée $\left(t<t_{0}\right)$, la force $f_{\mathrm{e}}(t \gg \tau)$ est proportionnelle à la viscosité statique [2] : $\eta=\int_{0}^{t \geqslant \tau} G\left(t^{\prime}\right) \mathrm{d} t^{\prime}$. A l'arrêt du déplacement $\left(t \geqslant t_{0}\right)$, $f_{\mathrm{e}}$ relaxe vers une valeur stationnaire nulle (Figs. 2a et $2 b)$. On a donc

$$
\left|f_{\mathrm{m}}\right|=\phi \eta r v \text { pour } t<t_{0} .
$$

Expérimentalement, lorsque l'éprouvette est déplacée à vitesse constante $v$, une mesure en régime stationnaire de l'intensité du courant circulant dans la bobine magnétique permet de déterminer la viscosité statique

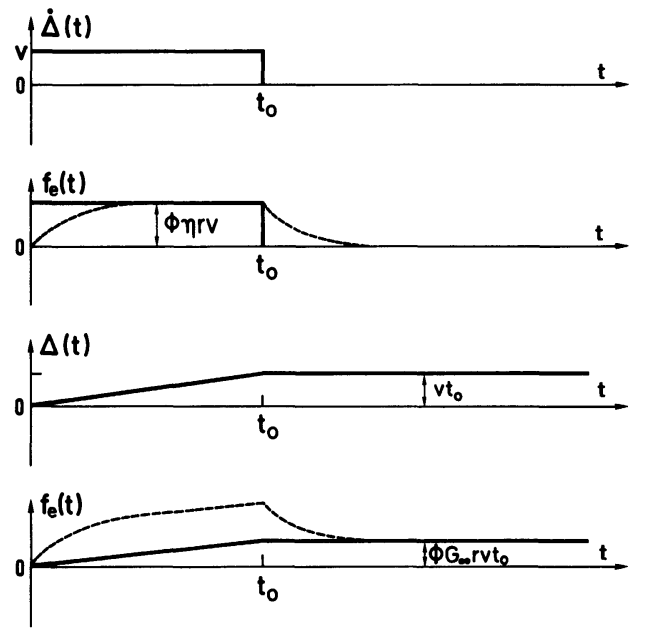

Fig. 2. - a) Vitesse de déplacement imposée à l'éprouvette. b) Force viscoélastique (extérieure) $f_{\mathrm{e}}$ à laquelle est soumise la bille magnétique lors du déplacement de l'éprouvette contenant un fluide. Les traits continus et en pointillés représentent respectivement le cas d'un fluide Newtonien et d'un fluide viscoélastique. c) Déplacement imposé à l'éprouvette. d) $f_{\mathrm{e}}$ dans le cas d'un solide élastique (trait continu) et dans le cas d'un solide viscoélastique (trait en pointillés).

[a) Displacement speed of the sample. b) External viscoelastic force $\left(f_{\mathrm{e}}\right)$ to which the magnetic sphere is submitted when the fluid sample is displaced. The full and dashed lines represent a Newtonian fluid and a viscoelastic fluid, respectively. c) Imposed sample displacement. d) External viscoelastic force $\left(f_{\mathrm{e}}\right)$ for the case of an elastic solid (full line) and a viscoelastic solid (dashed line).] 
d'un fluide viscoélastique si le taux de cisaillement réduit $\tau \dot{s}$ est faible.

- Cas d'un solide viscoélastique.

Dans le cas d'un solide viscoélastique le module de relaxation $G(t)$ est la superposition d'un module de fluide viscoélastique et d'un module de corps purement élastique [2]. Considérons d'abord le cas d'un corps purement élastique. En portant (11) dans (13) et (14) il vient :

$$
\begin{array}{lll}
f_{\mathrm{e}}(t)=\phi r G_{\infty} v t & \text { pour } t<t_{0} \\
f_{\mathrm{e}}(t)=\phi r G_{\infty} v t_{0} & \text { pour } t \geqslant t_{0} .
\end{array}
$$

Dans le cas d'un corps viscoélastique, la force $f_{\mathrm{e}}$ est donc la somme de la force calculée dans le paragraphe précédent et de celle calculée ci-dessus. A l'arrêt du déplacement $t \geqslant t_{0}$ la force $f_{\mathrm{e}}(t)$ relaxe vers une valeur stationnaire $f_{\mathrm{e}}(t \gg \tau)$ proportionnelle au module élastique statique $G_{\infty}$ de cisaillement (Figs. 2c et 2d) :

$$
\left|f_{\mathrm{m}}\right|=\phi G_{\infty} r v t_{0} .
$$

Expérimentalement, à l'arrêt du déplacement de l'éprouvette, une mesure de l'intensité du courant circulant dans la bobine magnétique permet de déterminer le module de cisaillement à fréquence nulle et faible déformation $\left(v t_{0} / r \ll 1\right)$.

1.2.2.2 Mesures en régime transitoire. - Considérons le cas d'un fluide viscoélastique, qui dans la gamme de temps qui nous intéresse $\left(\gtrsim 10^{-2} \mathrm{~s}\right)$ peut être représenté par un modèle de Maxwell à un seul temps de relaxation $\tau$ :

$$
G(t)=G_{0} \mathrm{e}^{-t / \tau}
$$

En portant l'équation (17) dans les équations (13) et (14) on a :

$$
\begin{array}{ll}
f_{\mathrm{e}}(t)=\phi r \eta v\left(1-\mathrm{e}^{-t / \tau}\right) & \text { pour } t<t_{0} \\
f_{\mathrm{e}}(t)=\phi r \eta v\left(1-\mathrm{e}^{-t_{0} / \tau}\right) \mathrm{e}^{-\left(t-t_{0}\right) / \tau} & \text { pour } t \geqslant t_{0} .
\end{array}
$$

Dans ces relations le paramètre $\phi$ est une constante purement géométrique uniquement en régime linéaire donc à faible taux de cisaillement réduit. Les distorsions dues à la fonction d'appareil du magnétorhéomètre sont négligeables (voir Eq. (A.16)) si $\hat{W}(p)$ est de l'ordre de 1 dans la gamme de fréquence $\mathrm{p}$, de l'ordre de quelques $\tau^{-1}$ où $\hat{f}_{\mathrm{e}}(p)$ est significativement différente de zéro. D'après l'expression (A.12) ceci correspond aux conditions suivantes :

$$
\tau_{\mathrm{m}}^{2} \tau_{\mathrm{i}} / \tau^{3} \ll 1 \quad \text { et } \quad \tau_{\mathrm{v}} \tau_{\mathrm{i}} / \tau^{2} \ll 1 .
$$

Pour réduire l'influence de la fonction d'appareil sur la réponse il est nécessaire de diminuer la constante d'intégration et d'augmenter la constante de rappel $K\left(\tau_{\mathrm{m}}^{2} \sim \tau_{\mathrm{v}} \sim 1 / K\right)$. La structure analytique exacte de $W(t)$ étant difficile à établir nous avons réalisé des simulations sur des exemples concrets (§ A.5). Un exemple de simulation est donné dans la figure 3 $\left(\tau_{\mathrm{m}}^{2} \tau_{\mathrm{i}} / \tau^{3}=1,9 \times 10^{-8}\right.$ et $\left.\tau_{\mathrm{v}} \tau_{\mathrm{i}} / \tau^{2}=1,8 \times 10^{-2}\right)$ le temps de relaxation $\bar{\tau}$ de $f_{\mathrm{m}}(t)$ est identique à $1 \%$ près au temps de relaxation $\tau$ de la force extérieure $f_{\mathrm{e}}(t)$. D'autre part les simulations montrent que, si les quantités $\tau_{\mathrm{m}}^{2} \tau_{\mathrm{i}} / \tau^{3}$ et $\tau_{\mathrm{v}} \tau_{\mathrm{i}} / \tau^{2}$ sont petites, la constante de temps de différentiation $\left(10^{-2}<\tau_{D}<10 \mathrm{~s}\right)$ n'affecte pas le temps de réponse $\bar{\tau}$.

Donc, à forte constante de rappel et faible constante de temps d'intégration, il est possible (avec un régulateur PID) de déterminer le temps de relaxation $\tau$ d'un corps viscoélastique.

\section{Description et caractéristiques du magnétorhéomè-} tre.

Dans ce paragraphe nous décrivons et donnons les caractéristiques de l'appareil (construit au centre de recherche du CEN Saclay) dont une photographie est donnée dans la figure 4.

\subsection{LÉVITATION ET PROFIL DU GRADIENT DE CHAMP} MAGNÉTIQUE. - Le gradient de champ magnétique nécessaire pour réaliser la lévitation est produit par une bobine magnétique $B$ (constituée d'un fil de cuivre de $\phi=0,3 \mathrm{~mm}$ ) dont la résistance est de $33 \Omega$ et l'inductance de $5,8 \times 10^{-2} \mathrm{H}$. La bille en samarium cobalt a son centre sur l'axe de symétrie vertical de la bobine (Fig. 5) elle n'est soumise qu'à une force magnétique axiale :

$$
f_{0 \mathrm{~m}}+f_{\mathrm{m}}=\frac{4 \pi}{3} r^{3} J \frac{\partial H_{x}\left(x^{\prime}\right)}{\partial x^{\prime}}
$$

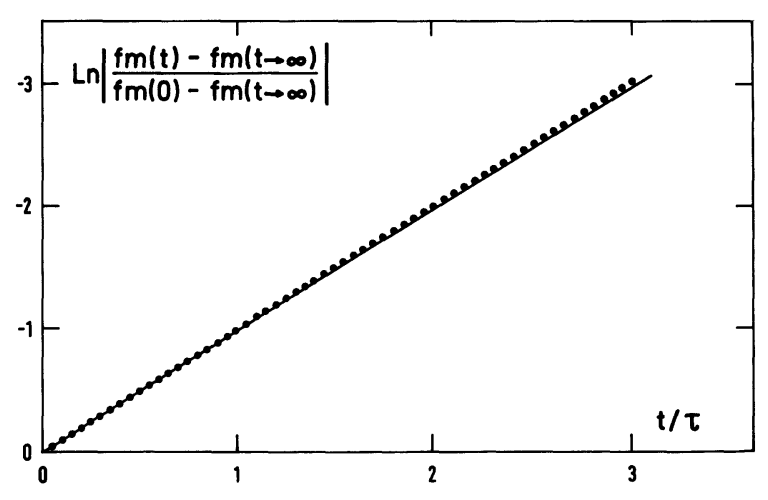

Fig. 3. - Simulation de l'expérience dans le cas d'un fluide viscoélastique de viscosité $4 \times 10^{3} \mathrm{P}$ et dont le temps de relaxation est $1 \mathrm{~s}$. Les constantes d'appareillage choisies sont : $\tau_{\mathrm{i}}=\tau_{\mathrm{D}}=10^{-2} \mathrm{~s}, \quad \alpha=10, \quad m=4 \times 10^{-3} \mathrm{~g}$, $r=5 \times 10^{-2} \mathrm{~cm}$ et $K=14 \alpha_{0}$ avec $\alpha_{0}=150$. Le trait plein représente la force extérieure $f_{\mathrm{e}}(t)$ et les points la réponse $f_{\mathrm{m}}(t)$.

[Simulation of an experiment in the case of a viscoelastic fluid having a viscosity of $4 \times 10^{3} \mathrm{P}$ and a relaxation time of $1 \mathrm{~s}$. The characteristics of the apparatus are : $\tau_{\mathrm{i}}=\tau_{\mathrm{D}}=10^{-2} \mathrm{~s}, \alpha=10, m=4 \times 10^{-3} \mathrm{~g}, r=5 \times 10^{-2} \mathrm{~cm}$, $\alpha_{0}=150$ and $K=14 \alpha_{0}$. The full line represents the external force $f_{\mathrm{e}}(t)$ and dots represent the response $f_{\mathrm{m}}(t)$.] 


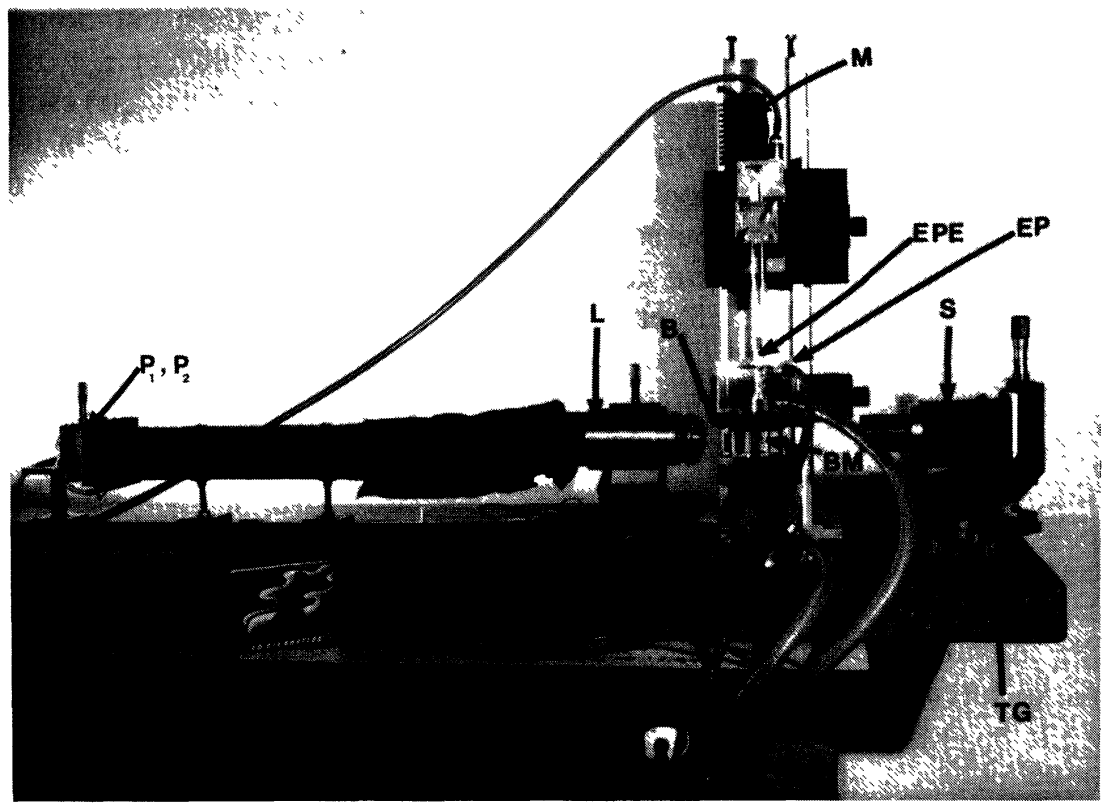

Fig. 4. - Photographie de l'appareil. E.P. enceinte en plexiglass. $T \rightarrow$ tuyaux permettant la circulation du liquide entre E.P. et le bain de thermalisation. Les autres lettres ont la même signification que dans la figure 1. L'ensemble du montage est réalisé sur une table en granit T.G. qui permet d'isoler l'appareil des vibrations du bâtiment.

[Photograph of the apparatus. E.P. = plexiglass countainer. T $\rightarrow$ Pipes for the circulation of the fluid between E.P. and the thermalization bath. The letters have the same meaning as in figure 1. The apparatus is set up on a granite table T.G. which prevents vibrations.]

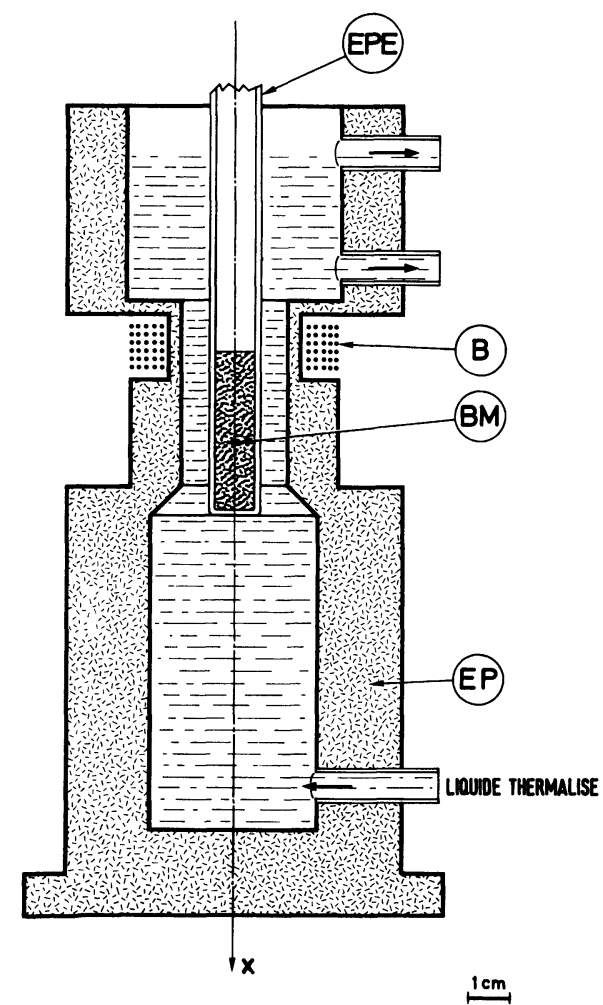

Fig. 5. - Géométrie de l'expérience. Les symboles ont la même signification que dans la figure 4 .

[Scheme of the plexiglass container. The symbols have same meaning as in figure 4 .]
$J$ est l'intensité d'aimantation de la bille $(\approx 660 \mathrm{G})$ et $x^{\prime}$ est la distance entre le centre de la bille et le bord inférieur de la bobine. Le gradient de champ magnétique $\partial H_{x}\left(x^{\prime}\right) / \partial x^{\prime}$ est proportionnel à l'intensité de courant, $I$, circulant dans la bobine :

$$
\frac{\partial H_{x}\left(x^{\prime}\right)}{\partial x^{\prime}}=n I P_{H}\left(x^{\prime}\right)
$$

$n$ est un facteur qui dépend de la géométrie de la bobine et du nombre de spires par unité de longueur. $P_{H}\left(x^{\prime}\right)$, le profil du gradient sur l'axe de la bobine est dans notre cas, en première approximation, linéaire (voir Fig. 6). En une position donnée, sur l'axe de la bobine, il existe toujours une valeur d'intensité de courant telle que la force magnétique s'exerçant sur la bille contrebalance toutes les autres forces appliquées à la bille : celle-ci l'évite.

2.2 ASSERVISSEMENT. - La source lumineuse est une lampe blanche (de puissance $<50 \mathrm{~W}$ ) alimenté par un générateur de tension stabilisée (Sodilec SDR 405). Grâce à cette source lumineuse et à une lentille, l'image de la bille est formée dans le plan $X, Y$ avec un grandissement $\gamma$ de l'ordre de 10 (voir Fig. 1). Les éclairements dans chacun des demi-plans $(X>0$ et $X<0)$ sont séparés à l'aide de deux prismes à réflexion totale $(\mathrm{p})$ et envoyés sur deux photodiodes à faible courant d'obscurité (Hewlett Packard 50824207). La tension de sortie, $u$, des photodiodes montées en pont permet de déterminer la position 


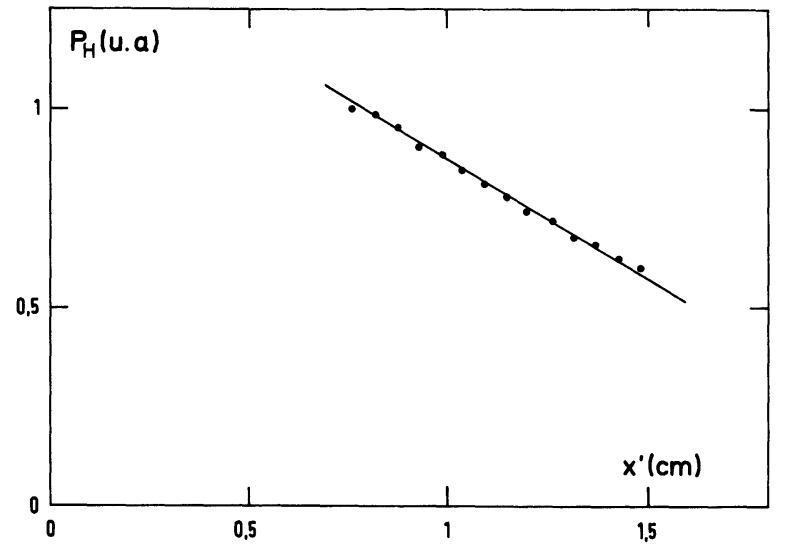

Fig. 6. - Variation du profil du gradient de champ magnétique $P_{H}$ en fonction de la distance, $x^{\prime}$, au bord inférieur de la bobine, on a $: \frac{\mathrm{d} P_{H} / \mathrm{d} x^{\prime}}{P_{H}} \lesssim 1,2 \mathrm{~cm}^{-1}$.

[Variation of the profil of the magnetic field gradient as a function of the distance $x^{\prime}$ from the lower edge of the coil. We have $\frac{\mathrm{d} P_{H} / \mathrm{d} x^{\prime}}{P_{H}} \lesssim 1.2 \mathrm{~cm}^{-1}$.

de la bille. Pour des positions voisines de l'origine nous avons :

$$
u=4 \beta E \gamma^{2} r x .
$$

$E$ représente le nombre de photons par unité de temps et de surface dans le plan $X, Y$. Le facteur $\beta$ dépend de la transmission optique du système, du rendement quantique et de la résistance interne des photodiodes.

La tension $u$, après une préamplification $\alpha_{0}$ variant de 1 à 1000 , est utilisée comme signal d'erreur de position : $\varepsilon=\alpha_{0} u$. Typiquement nous avons $\mathrm{d} \varepsilon / \mathrm{d} x \approx 6 \times 10^{-1} \quad \alpha_{0} \mathrm{~V} / \mathrm{cm}$. Le signal d'erreur $\varepsilon$ attaque le régulateur PID parallèle dont les caractéristiques sont les suivantes :

- action proportionnelle, le gain $\alpha$ varie de 1 à 11 ,

- action différentielle et intégrale, les constantes de temps varient de $10^{-2} \mathrm{~s}$ à $11 \mathrm{~s}$.

La tension de sortie du régulateur, $u_{\mathrm{s}}$, pilote l'alimentation en courant $I$ de la bobine magnétique. La constante de rappel globale de l'ensemble de la boucle d'asservissement s'exprime, en fonction des différents paramètres, comme suit :

$$
K=\frac{16 \pi}{3} \beta E \gamma^{2} r^{4} \alpha_{0} J n P_{H} I / u_{\mathrm{s}}
$$

Typiquement $K$ est de l'ordre $14 \alpha_{0}$. La partie électronique de l'asservissement a été réalisée par la compagnie ATNE.

2. 3 DÉPlaCEMENT DE L'ÉPROUVETTE. - L'éprouvette contenant l'échantillon est fixée sur une platine de translation entraînée par un moteur pas à pas (Microcontrôle UT100 50pp et M50-16). L'incrément de déplacement $\Delta x$ est de $0,1 \mu \mathrm{m}$ ou de $1 \mu \mathrm{m}$. La tension d'alimentation nécessaire au moteur est délivrée sous forme d'impulsions de fréquence $v$ (Microcontrôle IT5DCA1). La vitesse de déplacement de l'éprouvette $(v=v \Delta x)$ est déterminée en mesurant la périodicité $v^{-1}$ des impulsions (Fluke 7250A). La précision sur la détermination de la vitesse est de l'ordre de $10^{-3}$. Un cycle de déplacement à vitesse constante $(v)$ peut être prédéterminé :

- déplacement $\Delta_{0}$ pendant un temps $t_{0}\left(\Delta_{0}=v t_{0}\right)$,

- arrêt durant un temps $t_{\mathrm{a}}$,

- déplacement $-\Delta_{0}$ pendant un temps $t_{0}$

$\left(-\Delta_{0}=-v t_{0}\right)$

- arrêt durant un temps $t_{\mathrm{a}}$.

Avec ce système de déplacement il est réalisé :

- des vitesses $v$ allant de $2 \times 10^{-1} \mathrm{~cm} \mathrm{~s}^{-1}$ à $1,4 \times 10^{-6} \mathrm{~cm} \mathrm{~s}^{-1}$,

- des déplacements $\Delta_{0}$ compris entre $10^{-5} \mathrm{~cm}$ et $4 \mathrm{~cm}$,

- des arrêts dont la durée, $t_{\mathrm{a}}$, est de $10 \mathrm{~s}$ au minimum et de $3 \mathrm{~h}$ au maximum.

2.4 RÉGUlATION EN TEMPÉRATURE. - L'éprouvette est placée à l'intérieur d'une enceinte en plexiglass (Fig. 5) où circule un fluide thermalisé qui est soit de l'eau, soit un mélange eau/éthylène glycol. La gamme de température accessible est comprise entre $-30^{\circ} \mathrm{C}$ et $+70^{\circ} \mathrm{C}$. L'eau est thermalisée à l'aide d'un bain Tamson TXZ/250. Dans le cas du mélange eau/ éthylène-glycol la thermalisation est effectuée à l'aide d'un bain dont la régulation en température est assurée par une résistance chauffante et un thermoplongeur frigorifique (Fridigyn) asservis par un système de régulation par tout ou rien. La température de l'échantillon est contrôlée et homogène à mieux que $1 / 10^{\circ} \mathrm{C}$.

2.5 MESURES DES FORCES VISCOÉLASTIQUES. - L'intensité du courant, circulant dans la bobine, est déterminée en mesurant la différence de potentiel $U$ aux bornes d'une résistance $(R)$ en série avec la bobine magnétique (Fig. 1). $U$ est mesuré simultanément sur un enregistreur (Sefram BPD) un voltmètre digital (Fluke 8810A) et un analyseur multicanal (Intertechnique SA41). L'enregistreur permet de visualiser les forces viscoélastiques au cours du temps lorsque le cycle de déplacement décrit au paragraphe 2.3 est effectué (Fig. 7). Le voltmètre digital, interfacé à un calculateur (Hewlett-Packard 9820A) ne mesure la différence de potentiel que dans les régimes stationnaires. Les variations $\delta U_{\mathrm{s}}$ (voir Fig. 7) permettent de déterminer les caractéristiques viscoélastiques statiques (voir Eqs. (15) et (16)) :

$$
\delta U_{\mathrm{s}}\left(\propto f_{\mathrm{m}}\right)=C \phi \eta v \Delta x
$$

pour un fluide viscoélastique et

$$
\delta U_{\mathrm{s}}=C \phi G_{\infty} \Delta_{0}
$$

pour un solide viscoélastique.

$C$ est un facteur de calibration (voir $\S 3.1$ ). L'analyseur multicanal reproduit la variation de $U$ durant 


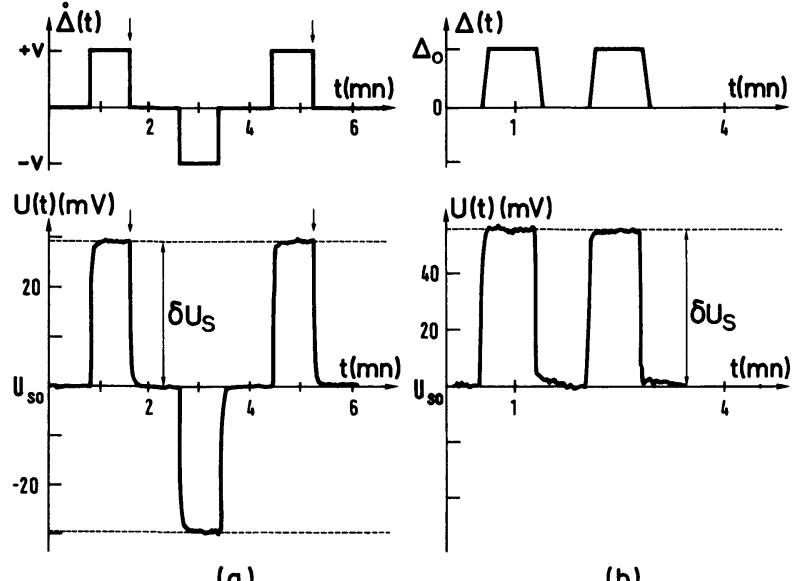

(a)

(b)

Fig. 7. - Exemples de variation de la tension $U(t)$ aux bornes de la résistance $R$ (Fig. 1) lorsqu'un cycle de déplacement est effectué : (a) dans le cas d'un fluide viscoélastique qui est une solution de polymères enchevêtrés polystyrènebenzène. La vitesse de déplacement $v=1,034 \times 10^{-3} \mathrm{~cm} / \mathrm{s}$. Les flèches $\downarrow$ indiquent les instants où l'ordre d'accumulation est donné au multicanal, (b) dans le cas d'un solide viscoélastique qui est une gélatine. Le déplacement total $\Delta_{0}=v t_{0}$ vaut $1 \mu \mathrm{m}$.

[Variation of the voltage accross the resistance $R$ (see Fig. 1) during a displacement cycle : (a) for a fluid viscoelastic, entangled polymer solution (polystyrene-benzene) the speed is $v=1.034 \times 10^{-3} \mathrm{~cm} \mathrm{~s}^{-1}$. The arrows indicate the times at which the accumulation on the multichannel analyser start, (b) for a viscoelastic solid (gelatine). The total displacement is $\Delta_{0}=v t_{0}=1 \mu \mathrm{m}$.]

les régimes transitoires. Le signal $U$ est échantillonné périodiquement à des intervalles de temps $\Delta t$ $(10 \mu \mathrm{s} \leqslant \Delta t \leqslant 8 \mathrm{~s})$ et stocké dans des mémoires (400). L'ordre de déclenchement de l'accumulation est donné au multicanal à l'arrêt du moteur lorsque la vitesse de déplacement de l'éprouvette passe d'une valeur positive $(+v)$ à une valeur nulle (voir Fig. 7). L'erreur de synchronisation entre le début d'accumulation et l'arrêt du moteur est inférieure à $10^{-4} \mathrm{~s}$. Après plusieurs accumulations le contenu des mémoires est envoyé dans le calculateur. Le profil expérimental $U(t)$ est ajusté à une fonction de la forme :

$$
U(t)=\tilde{A}+\tilde{B} \mathrm{e}^{-t / \tau} .
$$

Les paramètres $\tilde{A}, \tilde{B}$ et $\bar{\tau}$ sont calculés par une méthode de moindre carré. Le temps de relaxation $\bar{\tau}$ est le temps de relaxation viscoélastique si la fonction d'appareil est négligeable $(\S 1.2 .2 .2)$.

\section{Tests de l'appareillage.}

3.1 MesURE DE LA VISCOŚITÉ NEWTONIENNE STATIQUE. Pour déterminer la valeur absolue de cette viscosité il est nécessaire :

- de déterminer les conditions pour lesquelles les effets dus au taux de cisaillement et à la taille finie de l'échantillon sont négligeables,
- d'étalonner la variation de différence de potentielle $\delta U_{\mathrm{s}}$.

3.1.1 Influence du taux de cisaillement $\dot{s}$. - A chaque mesure il est vérifié que les écarts de différence de potentiel en régime stationnaire $\delta U_{\mathrm{s}}$ varient linéairement avec la vitesse, $v$, de déplacement de l'éprouvette. Un exemple de cette linéarité est donné dans la figure 8. Le paramètre important est le taux de cisaillement réduit $\dot{s} \tau, \dot{s}-$ par convention - est égal à $v / r$ et $\tau$ est le temps de relaxation viscoélastique le plus long du fluide. L'ensemble des mesures effectuées sur cet appareil ont montré que la force visqueuse $\left(\propto \delta U_{\mathrm{s}}\right)$ est mesurée avec une précision de $1 \%$ et n'est pas sensible aux taux de cisaillement si $\dot{s} \tau \lesssim 2 \times 10^{-2}$. Les taux de cisaillement appliqués ici sont plus faibles que ceux $\dot{s}_{\mathrm{t}}$ réalisés dans le cas d'un viscosimètre à bille tombante [1]. Si les billes sont identiques, dans les deux expériences, on a la relation suivante :

$$
\dot{s} / \dot{s}_{\mathrm{t}}=\delta U_{\mathrm{S}} / U_{\mathrm{os}},
$$

où $U_{0 S}$ représente la différence de potentiel nécessaire pour contrebalancer la force de gravitation effective. Typiquement $\delta U_{\mathrm{s}} U_{\mathrm{os}}$ est compris entre $5 \times 10^{-4}$ et $5 \times 10^{-2}$. Le magnétorhéomètre à bille présente donc l'avantage d'appliquer des taux de cisaillement faibles et variables ce qui permet de vérifier que la viscosité mesurée est bien Newtonienne.

3.1.2 Influence de la taille finie de l'échantillon. Les paramètres qui permettent de caractériser les effets de taille sont les dimensions réduites $2 r / d$, $2 r / l$ et $2 r / l^{\prime}$. $d$ représente le diamètre interne de l'éprouvette, $l$ et $l$ ' sont respectivement les distances

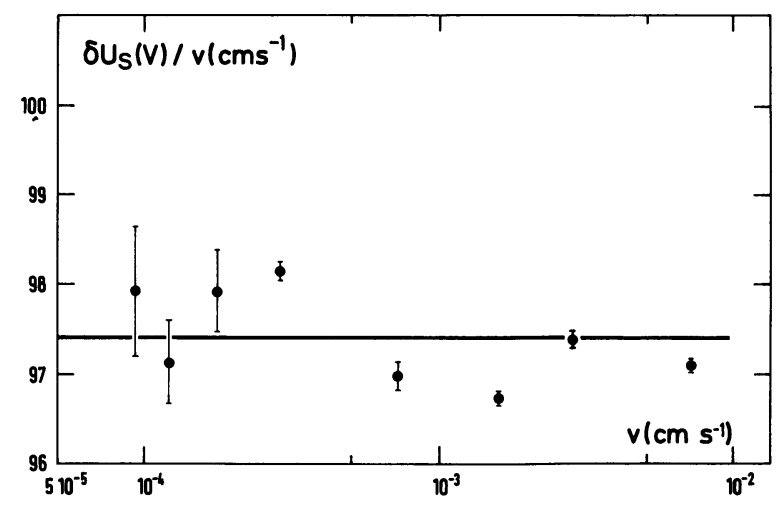

Fig. 8. - Exemple de test de proportionnalité entre les écarts de différence de potentiel en régime stationnaire $\left(\delta U_{\mathrm{s}}\right)$ et la vitesse de déplacement $v$ de l'éprouvette dans le cas d'un fluide viscoélastique. Solution de polymères enchevêtrés de viscosité $300,8 \mathrm{P}$ et dont le temps de relaxation le plus long est de $0,106 \mathrm{~s}$. Le taux de cisaillement réduit pour une vitesse de $10^{-2} \mathrm{~cm} / \mathrm{s}$ est de $2,2 \times 10^{-2}$.

[Check of the proportionality between the voltage difference and the speed $v$ of displacement, in the stationary regime for a viscoelastic fluid. Entangled polymer solution having a longest relaxation time of $0.106 \mathrm{~s}$. The reduced shear rate is $2.2 \times 10^{-2}$ at a speed of $10^{-2} \mathrm{~cm} \mathrm{~s}^{-1}$.] 
du centre de la bille à l'interface liquide-air et au fond de l'éprouvette. Pour un diamètre $d$ donné si $2 r / l$ et $2 r / l^{\prime} \lesssim 0,2, \phi$, défini par l'équation (9), est une constante. Dans cette région, la mesure de viscosité n'est pas sensible aux conditions aux limites, liquide/ air et liquide/fond de l'éprouvette. Elle dépend par contre du diamètre de l'éprouvette. L'influence du paramètre $2 r / d$ a été déterminée en faisant des mesures à faibles taux de cisaillement réduits dans des éprouvettes de différents diamètres pour un fluide Newtonien et un fluide viscoélastique. La variation de $\phi$ en fonction de $2 r / d$ (Fig. 9) montre que $\phi$ est indépendant du fluide, c'est un facteur purement géométrique car les mesures sont effectuées en régime stationnaire et à faibles taux de cisaillement réduits.

3.1.3 Etalonnage et domaine de mesure. - Le facteur de calibration $C \phi$ est déterminé à partir de la relation suivante :

$$
C \phi=\left(\frac{\delta U_{\mathrm{s}}}{\Delta x}\right)_{\mathrm{c}} \times \frac{1}{\eta_{\mathrm{c}}}
$$

en utilisant une huile au silicone de viscosité connue $\eta_{\mathrm{c}}$. Pour une bille et une bobine magnétique données le facteur $C \phi$ dépend du diamètre de l'éprouvette et de la position d'équilibre de la bille sur l'axe de la bobine. Usuellement les mesures sont effectuées avec une bille de diamètre $2 r=0,098 \mathrm{~cm}$ et des éprouvettes dont le diamètre moyen est $d=0,98 \mathrm{~cm}$ avec une tolérance de $\pm 0,01 \mathrm{~cm}$. Cette variation du

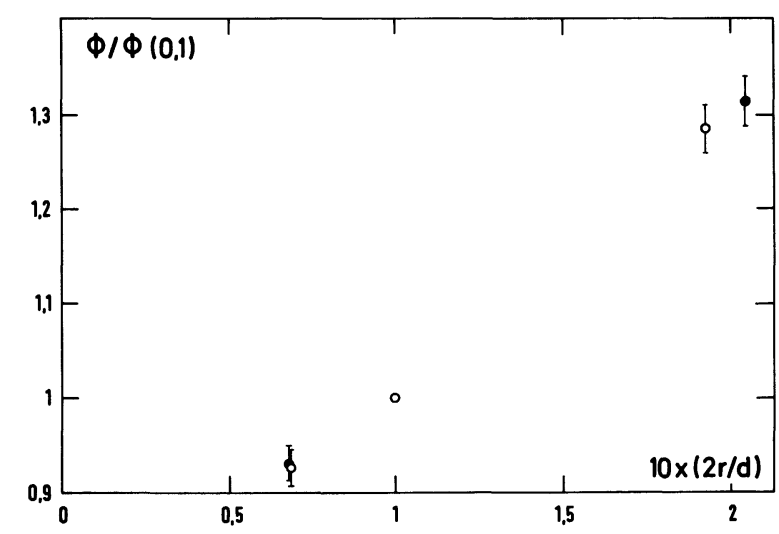

Fig. 9. - Influence des conditions aux limites. Variation en fonction du diamètre, $d$, de l'éprouvette du facteur $\phi$ normalisé par sa valeur à $2 r / d=0,1$ (pour $2 r / l^{\prime}$ et $2 r / l \leqslant 0,2$ ). $\phi$ Mesures sur un fluide Newtonien (huile au silicone $\eta=41,6 \mathrm{P}$ ). $\phi$ Mesures sur un fluide viscoélastique. (Polydiméthyl-siloxane, $\eta=4,70 \times 10^{3} \mathrm{P}$ et $\left.\tau=5 \times 10^{-2} \mathrm{~s}\right)$. La valeur absolue de $\phi$ est $6 \pi \times(0,98 \pm 0,05)$ pour $2 r / d=6,84 \times 10^{-2}$.

[Influence of boundary conditions. Variation as a function of the diameter of the quantity $\phi$ normalized by its value at $2 r / d=0.1\left(2 r / l^{\prime}\right.$ and $\left.2 r / l \leqslant 0.2\right)$. $\$$ Measurements on a Newtonian fluid (silicone oil $=41.6 \mathrm{P}$ ). $\phi$ Measurements on a viscoelastic fluid (Polydimethylsiloxane $\eta=4.7 \times 10^{3} \mathrm{P}$, $\left.\tau=5 \times 10^{-2} \mathrm{~s}\right)$. The absolue value of $\phi / 6 \pi$ is $0.98 \pm 0.05$ at $2 r / d=6.84 \times 10^{-2}$.] diamètre $d$ conduit à une imprécision de $\pm 0,1 \%$ sur le facteur de calibration $C \phi$. Pour tester cette calibration, des mesures ont été effectuées sur du dibutylphtalate, elles sont en accord avec celles obtenues avec un viscomètre à capillaire (voir tableau en appendice). Cette calibration peut être utilisée pour des liquides viscoélastiques uniquement si les mesures sont effectuées en régime stationnaire et à faibles taux de cisaillement.

Le magnétorhéomètre à bille permet de mesurer des viscosités allant de $3 \times 10^{-2}$ à $10^{5} \mathrm{P}$. La précision est d'autant meilleure que la viscosité est élevée, pour des viscosités supérieures ou de l'ordre de $50 \mathrm{P}$ la valeur absolue de la viscosité est déterminée avec une précision de $2 \%$.

3.2 Mesure de L'Élasticité STATiQue. - La bille magnétique doit être enrobée dans l'échantillon à étudier, ceci est réalisable, par exemple, au cours d'une gélification où la bille est maintenue en lévitation en phase liquide.

Pour de faibles déplacements $\Delta_{0}$ de l'éprouvette, les écarts de différences de potentiel en régime stationnaire varient linéairement avec le déplacement (Fig. 10). Le module élastique, $G_{\infty}$, ainsi mesuré est indépendant du cisaillement $: s=\Delta_{0} / r\left(2 \times 10^{-1} \gtrsim\right.$ $\left.s \geqslant 10^{-4}\right)$. Par raison de continuité et par analogie entre les équations d'hydrodynamique et d'élasticité linéaires, on peut penser que les effets de conditions aux limites en régime stationnaire à faible cisaillement sont identiques à ceux constatés dans le cas d'un fluide. Cette conjecture devra être vérifiée expérimentalement. La valeur absolue de $G_{\infty}$ est déterminée par une calibration identique à celle décrite précédemment.

Avec le magnétorhéomètre à bille il est possible de déterminer avec une précision de $10 \%$, des modules élastiques compris entre $10^{-2}$ dyne $/ \mathrm{cm}^{2}$ et $10^{4}$ dynes/ $\mathrm{cm}^{2}$.

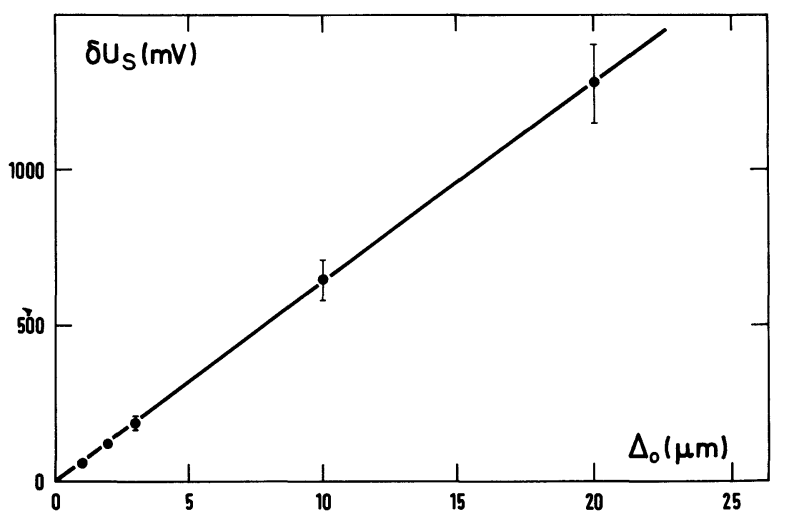

Fig. 10. - Exemple de variation des écarts de différence de potentiel en régime stationnaire $\left(\delta U_{\mathbf{s}}\right)$ en fonction du déplacement $\Delta_{0}$ de l'éprouvette dans le cas d'un solide viscoélastique (gélatine).

[Check of the proportionality between the voltage differences $\left(\delta U_{\mathrm{S}}\right)$ and the displacement of the sample $\Delta_{0}$, in the case of a solid viscoelastic (gelatine gel).] 


\subsection{Mesure DU TEMPS DE RELAXATION D'UN FLUIDE} VISCOÉlASTIQUE. - L'influence de la fonction d'appareil est déterminée sur des solutions de polystyrènebenzène à des concentrations où les polymères sont enchevêtrés. Dans ce cas la force viscoélastique extérieure $f_{\mathrm{e}}(t)$, relaxe exponentiellement avec un temps caractéristique $\tau$ le plus long du système polymérique [8]. Tout se passe comme si nous avions un fluide viscoélastique de Maxwell à un seul temps.

Dans le cas d'un régulateur à action proportionnelle, si la force inertielle est négligeable, un calcul exact montre que la force de rappel magnétique relaxe exponentiellement avec un temps :

$$
\bar{\tau}=\tau+\tau_{\mathrm{v}} / \alpha \text { avec } \tau_{\mathrm{v}} \propto 1 / \alpha_{0}
$$

où $\alpha$ et $\alpha_{0}$ sont définis au paragraphe 2.2. Cette dépendance a été vérifiée sur un échantillon de viscosité $\eta=3,94 \times 10^{3} \mathrm{P}$ (Fig. 11). Une extrapolation à gain infini $\left(\alpha \alpha_{0} \rightarrow+\infty\right)$ conduit à un temps de relaxation $\tau=1,14 \mathrm{~s}$. Avec un régulateur à action proportionnelle de gain fini, le temps caractéristique mesuré est toujours plus grand que le temps de viscoélastique.

Dans le cas d'un régulateur à action proportionnelle différentielle et intégrale à grande constante de rappel $\left(K \approx 14 \alpha_{0}, \alpha_{0}>100\right)$ le profil de relaxation de la force magnétique $f_{\mathrm{m}}(t)$ est une fonction exponentielle (Fig. 12). Le temps caractéristique $\bar{\tau}(1,17 \mathrm{~s})$ indépendant de $\alpha_{0}$ et $\alpha$ (voir Fig. 11) est identique à $3 \%$ près au temps $\tau$ trouvé par extrapolation à gain infini dans le cas d'un régulateur à action

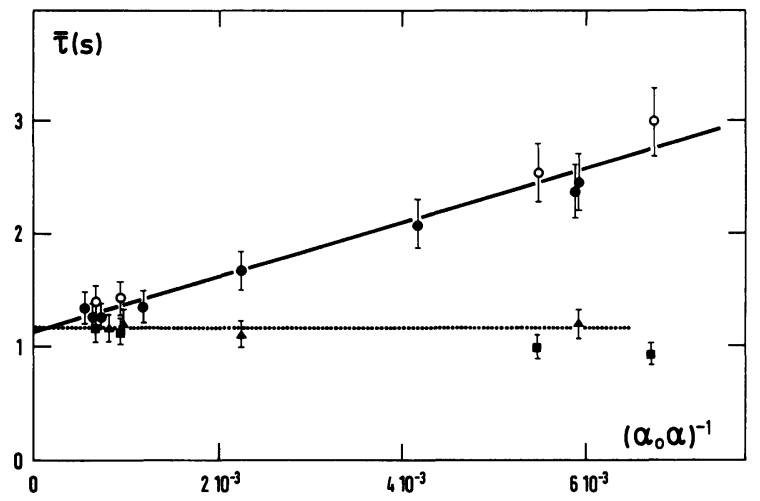

Fig. 11. - Temps de relaxation $\bar{\tau}$ de la force magnétique de rappel dans le cas d'un fluide viscoélastique. Les symboles $O$ et - représentent respectivement la variation de $\bar{\tau}$ en fonction de $\alpha_{0}$ et $\alpha$ lorsque le régulateur n'a qu'une action proportionnelle. Les symboles $\boldsymbol{\phi}$ et représentent les variations de $\bar{\tau}$ en fonction de $\alpha_{0}(\alpha=10,59=$ Cte $)$ et $\alpha$ $\left(\alpha_{0}=168,5=\right.$ Cte $)$ dans le cas d'une régulation PID $\left(\tau_{\mathrm{i}}=\tau_{\mathrm{D}}=10^{-2} \mathrm{~s}\right)$.

[Relaxation time $\bar{\tau}$ of the magnetic force $f_{\mathrm{m}}$ in the case of a viscoelastic fluids. The symbols $\bigcirc$ and $\bullet$ represent the variation of $\bar{\tau}$ as a function of $\alpha_{0}$ and $\alpha$, respectively, the monitor acting in the proportional mode. The symbols \$ et $\downarrow$ represent the variations of $\bar{\tau}$ as a function of $\alpha_{0}$ $(\alpha=10.59=$ const. $)$ and $\alpha\left(\alpha_{0}=168.5=\right.$ const. $)$ respectively, the monitor acting in the proportional + integral + differential mode (PID, $\tau_{\mathbf{i}}=\tau_{\mathbf{D}}=10^{-2} \mathrm{~s}$ ). $]$

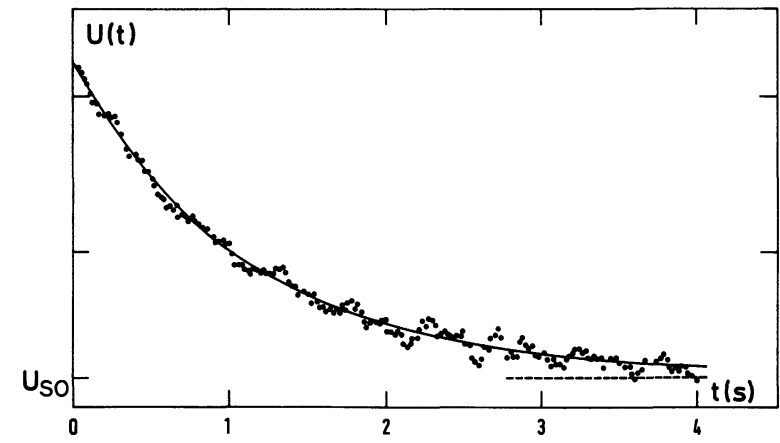

Fig. 12. - Exemple de relaxation de la force magnétique $(\propto U(t))$ pour une solution de polymères enchevêtrés dans le cas d'une régulation PID $\bullet$ points expérimentaux, la ligne continue représente le meilleur ajustement à une décroissance exponentielle.

[Example of relaxation of the magnetic force $(\propto U(t))$ in the case of entangled polymer solution with the PID monitor. The dots are the experimental points, the full line is the best fit corresponding to an exponential decay.]

proportionnelle. A forte valeur de la constante de rappel et à faible constante de temps d'intégration, le temps de relaxation mesuré est indépendant de l'action différentielle. Par exemple, pour un échantillon de viscosité $170 \mathrm{P}$ avec un gain de préamplification $\alpha_{0}=36$ on trouve que

$$
\begin{array}{lll}
\bar{\tau}=1,64 \pm 0,16 \mathrm{~s} & \text { si } & \tau_{\mathrm{D}}=5 \times 10^{-2} \mathrm{~s} \\
\bar{\tau}=1,61 \pm 0,16 \mathrm{~s} & \text { si } & \tau_{\mathbf{D}}=5 \mathrm{~s} .
\end{array}
$$

Le temps de relaxation $\bar{\tau}$ est également indépendant du taux de cisaillement réduit $\frac{v}{r} \bar{\tau}$ auquel est soumis le système polymérique lors des mesures

$$
\left(\frac{v}{r} \bar{\tau} \lesssim 2 \times 10^{-2}\right)
$$

Ces résultats expérimentaux sont en accord avec les résultats obtenus par simulation. Si le gain du préamplificateur est fort, et la constante de temps d'intégration faible, afin que $\frac{\tau_{\mathrm{m}}^{2} \tau_{\mathrm{i}}}{\tau^{3}} \ll 1$ et $\frac{\tau_{\mathrm{v}} \tau_{\mathrm{i}}}{\tau^{2}} \gg 1$, la force magnétique $f_{\mathrm{m}}(t)$ est une réplique dans le temps de la force extérieure $f_{\mathrm{e}}(t)$. Un régulateur à actions proportionnelle, différentielle et intégrale permet de mesurer directement le temps de relaxation viscoélastique. Une série de mesures montre que dans la configuration actuelle de l'appareil, des temps $\tau \gtrsim 4 \times 10^{-2} \mathrm{~s}$ sont mesurés avec une précision de $10 \%$.

\section{Conclusion.}

La sonde du magnétorhéomètre à bille étant de faible dimension, les mesures sont effectuées sur de petites quantités de produit $\left(\approx 1,5 \mathrm{~cm}^{3}\right)$. L'éprouvette contenant l'échantillon étant scellée les problèmes d'éva- 
poration, qui jouent un rôle important dans les solutions avec un solvant volatil, sont éliminés. La géométrie de l'appareil permet de thermaliser avec précision l'échantillon $\left( \pm 0,1^{\circ} \mathrm{C}\right)$. L'appareil décrit ici présente aussi l'avantage de pouvoir appliquer des cisaillements $\left(s \gtrsim 2 \times 10^{-4}\right)$ et taux de cisaillement $\left(3 \times 10^{-5} \mathrm{~s}^{-1} \lesssim \dot{s} \lesssim 4 \mathrm{~s}^{-1}\right)$ faibles et de pouvoir les faire varier aisément.

En régime stationnaire, les quantités statiques mesurées sont :

- la viscosité, avec une précision de $2 \%$ sur une gamme allant de $3 \times 10^{-2}$ à $10^{5} \mathrm{P}$

- le module élastique, avec une précision de $10 \%$ sur une gamme allant de $10^{-2}$ à $10^{4}$ dyne $/ \mathrm{cm}^{2}$.

En régime transitoire, dans des cas simples, le temps de relaxation viscoélastique le plus long est déterminé avec une précision de $10 \%$. Le plus court temps de relaxation mesurable est de l'ordre de $40 \mathrm{~ms}$.

Un appareil comme le magnétoréhomètre à bille est adapté à l'étude des propriétés viscoélastiques des solutions semi-diluées de polymère où sont déterminées les variations de la viscosité et du temps de relaxation le plus long en fonction de la concentration et du poids moléculaire des polymères [8-10]. Cet appareil est aussi adapté à l'étude de la transition solgel, où au cours de la gélification, la viscosité et l'élasticité dans le bain de réaction $[11,12]$ sont mesurés.

\section{Remerciements.}

Les auteurs tiennent à remercier M. Labouise pour son assistance technique et $\mathbf{J}$. M. Bernal pour sa collaboration dans les expériences de tests et de caractérisations de l'appareillage.

\section{Appendice}

La transformée de Laplace d'une quantité $A(t)$ est par définition [13] :

$$
\hat{A}(p)=\int_{0}^{\infty} \mathrm{e}^{-p t} A(t) \mathrm{d} t
$$

1. Fonction d'appareil, $A$, du régulateur PID parallèle.

D'après l'équation (5) il vient :

$$
\hat{A}(p)=-K\left[\alpha+p \tau_{\mathrm{D}}+\frac{1}{p \tau_{\mathrm{i}}}\right] .
$$

\section{Constante de rappel généralisée $\hat{\mu}(p)$.}

D'après les équations (10) et (11) nous avons pour :

- un fluide Newtonien

$$
\hat{\mu}(p)=\phi \eta r,
$$

— un solide élastique

$$
\hat{\mu}(p)=\phi r G_{\infty} / p .
$$

D'après les équations (17) et (9) nous avons pour un fluide viscoélastique à un seul temps caractéristique

$$
\hat{\mu}(p)=\hat{\mu} / p=\frac{\phi r G_{0} \tau}{p \tau+1}=\frac{\phi r \eta}{p \tau+1} .
$$

Pour un solide viscoélastique qui est la superposition d'un fluide viscoélastique et d'un solide élastique [2] d'après (A.4) et (A.5) nous avons :

$$
\hat{\mu}(p)=\phi r\left(\frac{G_{0} \tau}{p \tau+1}+\frac{G_{\infty}}{p}\right) .
$$

\section{Fonctions d'appareil du magnétorhéomètre.}

D'après l'expression (12b) la fonction d'appareil du magnétorhéomètre, $\hat{W}$, est reliée à la fonction d'appareil de l'asservissement $\hat{A}$ par la relation suivante :

$$
\hat{W}=-\hat{A} . \hat{\Omega}
$$

avec

$$
\hat{\Omega}=\left[m p^{2}+p \hat{\mu}(p)-\hat{A}(p)\right]^{-1} .
$$

En utilisant les équations (A .2) à (A.7), il vient pour les différents cas les fonctions d'appareils suivantes :

- Fluide Newtonien

$$
\hat{W}(p)=\frac{p^{2} \tau_{\mathrm{i}} \tau_{\mathrm{D}}+p \alpha \tau_{\mathrm{i}}+1}{p^{3} \tau_{\mathrm{i}} \tau_{\mathrm{m}}^{2}+p^{2}\left(\tau_{\mathrm{i}} \tau_{\mathbf{D}}+\tau_{\mathrm{i}} \tau_{\mathrm{v}}\right)+p \alpha \tau_{\mathrm{i}}+1}
$$

où $\tau_{\mathrm{m}}$ et $\tau_{\mathrm{v}}$ sont définis comme suit :

$$
\tau_{\mathrm{m}}^{2}=m / K \quad \text { et } \quad \tau_{\mathrm{v}}=\phi \eta r / K
$$

— Solide élastique

avec

$$
\hat{W}(p)=\frac{p^{2} \tau_{\mathrm{i}} \tau_{\mathrm{D}}+p \alpha \tau_{\mathrm{i}}+1}{p^{3} \tau_{\mathrm{m}}^{2} \tau_{\mathrm{i}}+p^{2} \tau_{\mathrm{D}} \tau_{\mathrm{i}}+p \tilde{\alpha} \tau_{\mathrm{i}}+1}
$$

$$
\hat{\alpha}=\phi r G_{\infty} / K+\alpha
$$

— Fluide viscoélastique

$$
\hat{W}(p)=\frac{p^{3} \tau_{\mathrm{i}} \tau_{\mathrm{D}} \tau+p^{2}\left(\alpha \tau_{\mathrm{i}} \tau+\tau_{\mathrm{i}} \tau_{\mathrm{D}}\right)+p\left(\alpha \tau_{\mathrm{i}}+\tau\right)+1}{p^{4} \tau_{\mathrm{m}}^{2} \tau_{\mathrm{i}} \tau+p^{3}\left(\tau_{\mathrm{i}} \tau_{\mathrm{D}} \tau+\tau_{\mathrm{i}} \tau_{\mathrm{m}}^{2}\right)+p^{2}\left(\alpha \tau_{\mathrm{i}} \tau+\tau_{\mathrm{i}} \tau_{\mathbf{D}}+\tau_{\mathrm{i}} \tau_{\mathrm{v}}\right)+p\left(\alpha \tau_{\mathrm{i}}+\tau\right)+1}
$$

— Solide viscoélastique

$$
\hat{W}(p)=\frac{p^{3} \tau_{\mathrm{i}} \tau_{\mathbf{D}} \tau+p^{2}\left(\alpha \tau_{\mathrm{i}} \tau+\tau_{\mathrm{i}} \tau_{\mathbf{D}}\right)+p\left(\alpha \tau_{\mathrm{i}}+\tau\right)+1}{p^{4} \tau_{\mathrm{m}}^{2} \tau_{\mathrm{i}} \tau+p^{3}\left(\tau_{\mathrm{i}} \tau_{\mathbf{D}} \tau+\tau_{\mathrm{i}} \tau_{\mathrm{m}}^{2}\right)+p^{2}\left(\tilde{\alpha} \tau_{\mathrm{i}} \tau+\tau_{\mathrm{i}} \tau_{\mathbf{D}}+\tau_{\mathrm{i}} \tau_{\mathrm{v}}\right)+p\left(\tilde{\alpha} \tau_{\mathrm{i}}+\tau\right)+1} .
$$


Dans le cas où l'action intégrale est supprimée les fonctions d'appareils correspondantes à chaque type de corps étudié sont déduites des expressions (A.8) ou (A.10) ou (A.12) ou (A.13) en faisant tendre formellement $\left(p \tau_{\mathbf{i}}\right)$ vers l'infini. Si l'action différentielle est supprimée, les fonctions d'appareils sont obtenues en posant $\tau_{\mathrm{D}}=0$.

4. Relation entre force extérieure et magnétique en régime stationnaire.

Très généralement nous avons :

$$
\lim _{t \rightarrow \infty} f_{\mathrm{m}}(t)=\lim _{p \rightarrow 0} p \hat{f}_{\mathrm{m}}(p) .
$$

En utilisant la définition de la fonction d'appareil il vient la relation suivante :

$$
\lim _{t \rightarrow \infty} f_{\mathrm{m}}(t)=-\left[\lim _{p \rightarrow 0} \hat{W}(p)\right] \lim _{t \rightarrow \infty} f_{\mathrm{e}}(t) .
$$

Si le régulateur est un régulateur PID parallèle dans tous les cas $\lim _{p \rightarrow 0} \hat{W}(p)=1$, la force magnétique de rappel est directement opposée à la force extérieure.

\section{Principe de simulation du magnétorhéomètre en} régime transitoire.

D'après la définition de la fonction d'appareil Equation (2) on a :

D'après la définition de la fonction d'appareil (Eq. (2)) on a :

$$
-\hat{f}_{\mathrm{m}}(p)=\hat{W}(p) \hat{f}_{\mathrm{e}}(p)
$$

En général, quatre fréquences caractéristiques différentes $-W_{K}$ annulent le dénominateur de $\hat{W}(p)$. Dans ces conditions $\hat{W}(p)$ peut être décomposée en éléments simples :

$$
\hat{W}(p)=\sum_{K=1}^{4} \frac{A_{K}}{p+W_{K}}
$$

où $A_{K}$ sont des coefficients.
D'après (A.17) dans l'espace temps on $a$ :

$$
W(t)=\frac{1}{2} \sum_{K=1}^{4} A_{K} \mathrm{e}^{-W_{K} t}+A_{K}^{*} \mathrm{e}^{-W_{K}^{*} t},
$$

où $A_{K}^{*}$ et $W_{K}^{*}$ sont les complexes conjugués de $A_{K}$ et $W_{K}$. Les fréquences caractéristiques $-W_{K}$ et les coefficients $A_{K}$ sont déterminés numériquement. Pour évaluer les distorsions de la fonction d'appareil il est supposé qu'une force extérieure :

$$
f_{\mathrm{e}}(t) \sim \theta(t)\left(1-\mathrm{e}^{-t / \tau}\right),
$$

est appliquée à l'instant $t=0$.

La force magnétique correspondante est, d'après (A.18), (A.19) et (2), de la forme

$$
\begin{aligned}
\left|f_{\mathrm{m}}(t)\right|=\frac{1}{2} \sum_{K=1}^{4} \frac{A_{K}}{W_{K}} & \left(1-\mathrm{e}^{-W_{K} t}\right)+\frac{A_{K}^{*}}{W_{K}^{*}}\left(1-\mathrm{e}^{-W_{K}^{*} t}\right) \\
& +\frac{1}{2} \sum_{K=1}^{4} \frac{A_{K} \tau}{W_{K} \tau-1}\left(\mathrm{e}^{-W_{K} t}-\mathrm{e}^{-t / \tau}\right) \\
& +\frac{A_{K}^{*} \tau}{W_{K}^{*} \tau-1}\left(\mathrm{e}^{-W_{K}^{*} t}-\mathrm{e}^{-t / \tau}\right)
\end{aligned}
$$

La force $\left|f_{\mathrm{m}}(t)\right|$ est calculée numériquement à partir de l'expression (A.20) et comparée à la force extérieure

\begin{tabular}{|c|c|c|c|}
\hline$T^{\circ} \mathrm{C}$ & $\begin{array}{c}\eta(\mathrm{cp}) \\
\text { magnétorhéomètre }\end{array}$ & \multicolumn{2}{|c|}{$\begin{array}{c}\eta(\mathrm{cp}) \\
\text { capillaire }\end{array}$} \\
\hline 2 & $49,20 \pm 0,98$ & $49,57[6]$ & $49,40[7]$ \\
\hline 25 & $15,53 \pm 0,31$ & 15,81 & 15,80 \\
\hline 45 & $7,57 \pm 0,15$ & 7,85 & 7,69 \\
\hline
\end{tabular}
(A.19).

Tableau. - Valeurs absolues de la viscosité du dibutylphtalate obtenues avec le magnétorhéomètre à bille. A titre de comparaison sont reportées des valeurs obtenues avec des viscosimètres à capillaire $[6,7]$.

[Viscosity of the dibutylphtalate measured using the magnetic sphere rheometer. Values obtained using capillary viscometers are reported.]

Bibliographie

[1] EIRICH, F. R., Rheology, volumes 1, 2, 3 (Academic press, New York-London) 1960.

[2] Ferry, J. D., Viscoelastic properties of polymers (second edition, John Wiley and Sons, Inc. New York) 1970.

[3] Le symbole $*$ représente un produit de convolution, comme les fonctions (et opérateurs) utilisés sont définis pour $t \geqslant 0$, nous avons : $g(t) * h(t) \equiv$ $\int_{0}^{t} g\left(t-t^{\prime}\right) h\left(t^{\prime}\right) \mathrm{d} t^{\prime}$

[4] $\dot{y}(t)$ représente la dérivée par rapport au temps de la quantité $y(t): \dot{y}(t) \equiv \frac{\mathrm{d} y(t)}{\mathrm{d} t}$.

[5] Gordon, M., Hunter, S. C., Loue, J. A. Ward, T. C., Nature 217 (1968) 735.
[6] Stepaneck, P., Thèse Prague (Tchéchoslovaquie) 1981. [7] BRIED, E. M. et al., Ind. Eng. Chem. 39 (1947) 484.

[8] Adam, M., Delsanti, M., J. Physique 44 (1983) 1185.

[9] Adam, M., Delsanti, M., J. Physique Lett. 40 (1979) L-523.

[10] Adam, M., Delsanti, M., J. Physique 43 (1982) 549.

[11] Adam, M., Delsanti, M., J. Physique Lett. 40 (1979) L-539.

[12] Adam, M., Delsanti, M., Durand, D., Hild, G., Munch, J. P., Pure Appl. Chem. 53 (1981) 1489.

[13] Pour les propriétés des transformées de Laplace voir par exemple Schwartz, L. Méthodes mathématiques pour les sciences physiques (Hermann, Paris) 1956. 